\title{
Ganganalyse und funktionelle Anatomie
}

\author{
Thomas Mittlmeier, Magdalena Müller-Gerbl
}

\section{Zusammenfassung}

Neben der klassischen deskriptiven topographischen Anatomie und dem in vitro-Experiment stehen uns heute nicht invasive Verfahren der funktionellen Analyse in der Anatomie zur Verfügung, die Rückschlüsse auf die individuelle Lastverteilung am oberen Sprunggelenk und den Gelenken des Fußes erlauben (z.B. CT-Osteoabsorptiometrie) und konkrete Hinweise auf die Pathobiomechanik geben können. Auch aus dem breiten Repertoire ganganalytischer Untersuchungstechniken können wir bei der Analyse von Abrollstörungen bei Erkrankungen oder nach Verletzungen des Fußes auf standardisierte, rasch einsetzbare und zuverlässige Methoden zurückgreifen. Während spezifische Fragestellungen nach wie vor den aufwendigen Einsatz kombinierter kinetisch-kinematischer Ganganalyseverfahren erforderlich machen, bietet die dynamische Druckverteilungsmessung unter dem Fuß oder im Schuh beim Gehen die Möglichkeit, Funktionsstörungen untersucherunabhängig quantitativ zu erfassen, Hinweise auf die gestörte Mechanik nahe dem Messort zu erhalten und therapeutische Maßnahmen auf ihre Effizienz hin zu untersuchen. Die moderne Ganganalyse bietet dem Kliniker neben prognostisch relevanten Aspekten eine Hilfe beim objektiven Vergleich konkurrierender therapeutischer Verfahren, der Qualitätskontrolle nach Therapie und zum Funktionsscreening vor und nach geplanten rekonstruktiven Eingriffen.

OP-JOURNAL 2001; 17: 4-13

(C) Georg Thieme Verlag Stuttgart · New York

\section{Einleitung}

Klassisch anatomische Betrachtungen analysieren die jeweiligen Strukturen des Fußes isoliert voneinander. Ausschließlich statische Betrachtungen der anatomischen Strukturen können dann zu weitreichenden Fehlschlüssen führen, deren bekanntestes Beispiel die Annahme einer Längs- und Quergewölbestruktur des Fußes mit Dreipunktauflage ist (Kalkaneus - Metatarsale I und -V-Köpfchen). Bereits Virchow hat dieses Konzept vor 100 Jahren als falsch verworfen, es findet sich jedoch weiterhin hartnäckig auch in Lehrbüchern aus neuerer Zeit wieder [9] (Abb.1).

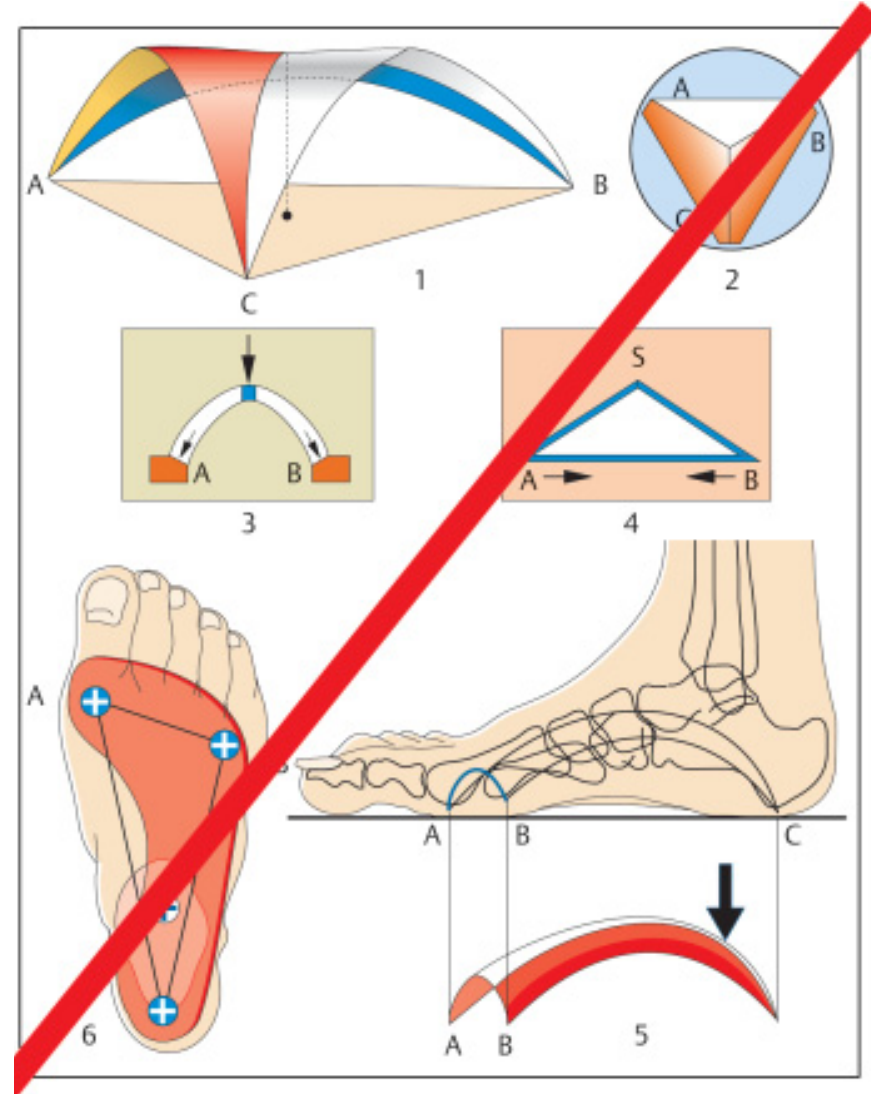

Druckverteilungsmessungen unter dem Fuß im Stand und beim Gehen belegen jedoch, dass die Metatarsalia II-V gleichermaßen belastet werden und die Drucke unter dem Metatarsaleköpfchen III höher sind als unter den Metatarsaliaköpfchen II und IV [8].

Für das elementare Verständnis so komplexer Vorgänge wie des Gehens ist deshalb die Kenntnis des Zusammenspiels der funktionellen Einheiten des Fußes entscheidend.

Die Analyse der Körperbewegungen wird im allgemeinen als Kinesiologie bezeichnet. Berücksichtigen wir nur die Bewegungen in den Gelenken ohne Beachtung der wirksamen Kräfte, so wird dies unter dem Begriff der Kinematik zusammengefasst.

Abb.1 Die „Tripod theorie" der Belastung des Fußes beim Gehen und Stehen (Kalkaneus-Metatarsaliaköpfchen I und V) ist mit ganganalytischen Verfahren eindeutig widerlegbar. 
So sind die Bewegungen der Tibia, der Sprunggelenke und der Gelenke der Fußwurzel und des Vorfußes in typischer Weise miteinander gekoppelt (Abb.2 u.3). Da in der Literatur Bewegungen unterschiedlich definiert werden, soll in Übereinstimmung mit der Mehrzahl der Autoren als Eversion und Inversion die Bewegung um die schräg gestellte Achse des unteren Sprunggelenkes verstanden werden (Eversion $=$ Senkung des inneren Fußrandes, Inversion = Hebung des inneren Fußrandes), unter Pro- und Supination die Bewegung um die Fußlängsachse vor dem Chopartgelenk in der subtalaren Platte verstanden werden [7].

Die Bewegungskoppelung der Strukturen des Fußes ist von erheblicher Relevanz für die Kraftübertragung zwischen Rück- und Vorfuß, etwa beim Abrollvorgang, um definierte Gelenkregionen für die effektive Lasttransmission im Wechsel zu „sperren“ oder zu „entriegeln“ [20] (Abb. 2).
Wie aus der Abbildung klar zu erkennen ist, wird beim normalen Gehen nur ein kleiner Teil des anatomisch möglichen Bewegungsumfanges der jeweiligen Gelenke eingesetzt.

Die Analyse der Kräfte, die beim Gehen wirksam werden, bezeichnet man als Kinetik.

Die direkte nicht invasive Messung von Gelenk- und Muskelkräften ist nicht möglich. Man ist also auf indirekte Messungen der Komponenten der Bodenreaktionskraft (verursacht durch die Bewegung des Körperschwerpunkts) mit Hilfe von Kraftmessplatten oder die flächenauflösende Untersuchung der Vertikalkomponente der Bodenreaktionskraft und der Druckverteilung unter dem Fuß beim Gehen (Plattform- und Sohlenmessung) angewiesen.

Nur mit Hilfe entsprechender theoretischer Modelle des Fußes kann dann anhand der Prinzipien der inversen Dynamik aus definierten kinetischen und kinematischen Messungen in Kenntnis an- thropometrischer Parameter auf innere Gelenkkräfte und -momente geschlossen werden (mathematische Modellbildung) $[6,7,14,21,22]$.

\section{Funktionelle Anatomie und Biomechanik}

\section{Oberes Sprunggelenk}

Das obere Sprunggelenk (OSG) ist nur in erster Näherung ein Scharniergelenk. Die Gelenkflächen sind keinesfalls kongruent („physiologische Inkongruenz“), die Talusrolle ist ventral $3-5 \mathrm{~mm}$ breiter als dorsal. Bei Dorsalextension ist somit von einer geringfügigen Erweiterung der Sprunggelenksgabel von 1-2 mm auszugehen.

Die Form der Gelenkflächen ist dafür verantwortlich, dass stets nur Teile der Gelenkflächen die jeweilige Kraft aufnehmen, wobei die Lage und Größe der Kontaktflächen ebenso wie die Druckverteilung von der Gelenkstellung abhängig sind.

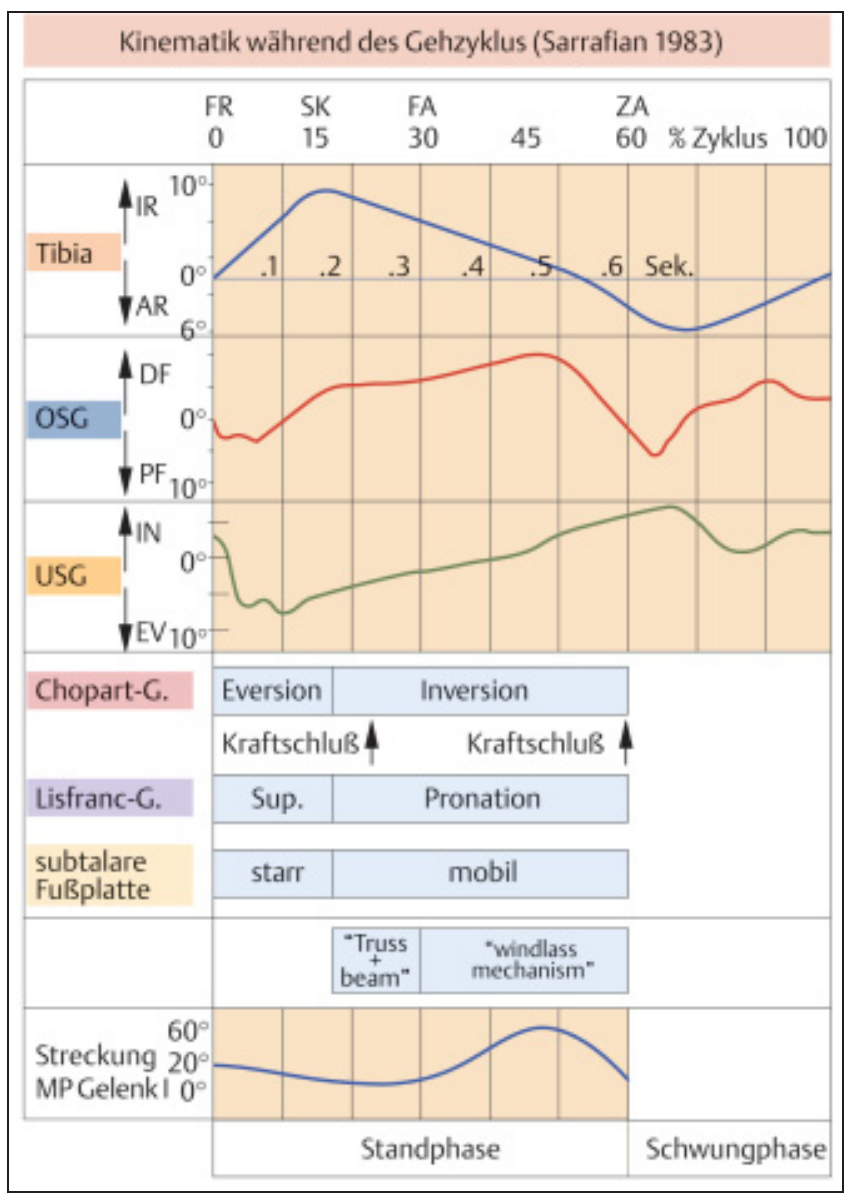

Abb.2 Kinematik der Sprunggelenke und des Fußes während des Gehzyklus. Prozesse der dynamischen Stabilisation des Fußes in der Standphase.

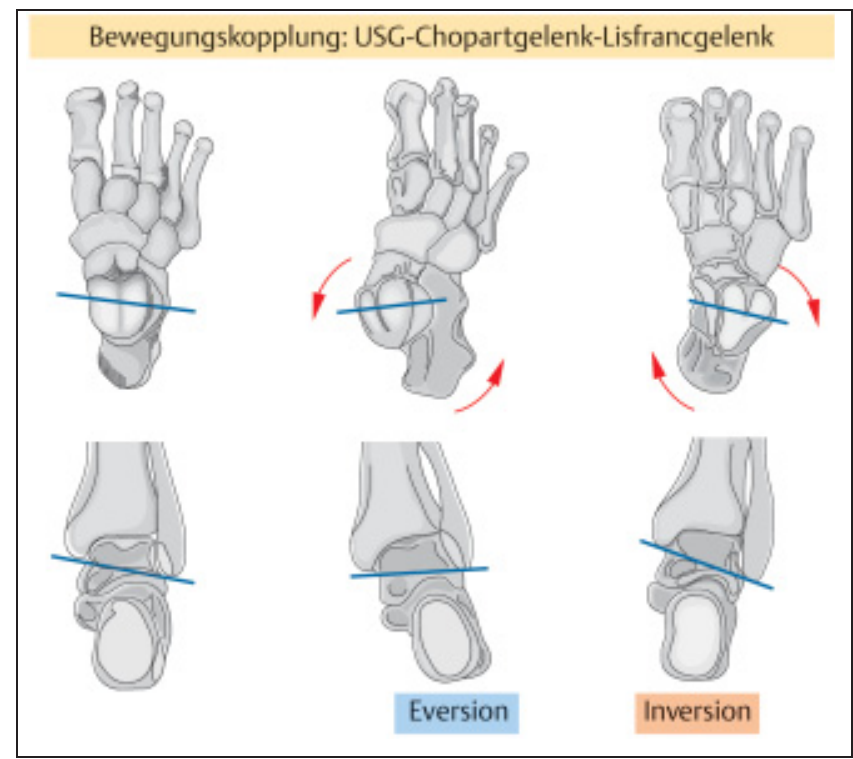

Abb.3 Die Bewegungen im unteren Sprunggelenk sind eng an diejenigen im Chopartgelenk gekoppelt und für die Kraftübertragung zwischen Rück- und Vorfuß während des gesamten Abrollens von fundamentaler Bedeutung. Die Eversion des Rückfußes während der frühen Standphase des Gehzyklus ist an eine Supination des Vorfußes, die folgende Inversion des Rückfußes an eine Pronation des Vorfußes gekoppelt. 
Im Ablauf von der Plantarflexion zur Dorsalextension wandert die momentane Kontaktfläche von den dorsalen Anteilen der Trochlea tali über eine zentrale Lage in Neutralnullposition ventralwärts, wobei ihre Größe zunimmt, während der auftretende Gelenkdruck sinkt. Bei Inversion kommt es zu einer Medialverschiebung, bei der Eversion zu einer Verlagerung der Kontaktfläche nach lateral. Mit steigender Gelenkkraft kann durch eine Vergrößerung der Kontaktläche ein annähernd gleichbleibender Gelenkdruck gehalten werden [16]. Unter Last treten Rotationsbewegungen und eine geringe $\mathrm{Di}$ stalverschiebung der Fibula auf.

Für die Gelenkfunktion ist das ungestörte dreidimensionale Bewegungsspiel im Fibula-Syndesmosenkomplex von elementarer Bedeutung [10].

Bei Gehbelastung treten Kräfte im oberen Sprunggelenk bis zum 3-5fachen des Körpergewichts auf [18]. Der rein axialen Druckbeanspruchung der Gelenkflächen durch die gelenkresultierende Kraft sind Zugkräfte seitens der Kollateralbänder überlagert.

Die Gelenkknorpeldicke ist keineswegs gleichmäßig verteilt, sondern zeigt erhebliche regionale Varianz. Diese weist ebenso wie die Verteilung der subchondralen Mineralisierung, die als morphologisches Korrelat der individuellen Beanspruchung eines Gelenks erachtet werden kann, typische Verteilungsmuster auf [16].

Üblicherweise ist beim jungen Menschen ein bizentrisches Muster zu beobachten: am Talus findet sich jeweils ein Dichtemaximum an der kantennahen lateralen und medialen Rolle korrespondierend mit der subchondralen Dichteverteilung an der distalen Tibiagelenkfläche (Abb. 4b). Dies bedeutet, dass der zentrale Gelenkbereich relativ zu den Kantenbereichen geringer belastet wird. Kommt es mit zunehmender Degeneration im Alter zu einer Abflachung der Trochleaführungsrinne, „verschmelzen“ die beiden Dichtemaxima zu einem monozentrischen Muster der subchondralen Dichte (Abb.4a). Dies kann so verstanden werden, dass die Gelenkkongruenz im Alter zunimmt, jedoch auf Kosten einer optimalen Druckverteilung. Dies kann dazu führen, dass bestimmte Gelenkflächenareale einem Dauerdruck ausgesetzt sind, der den Gelenkknorpel schädigen kann.

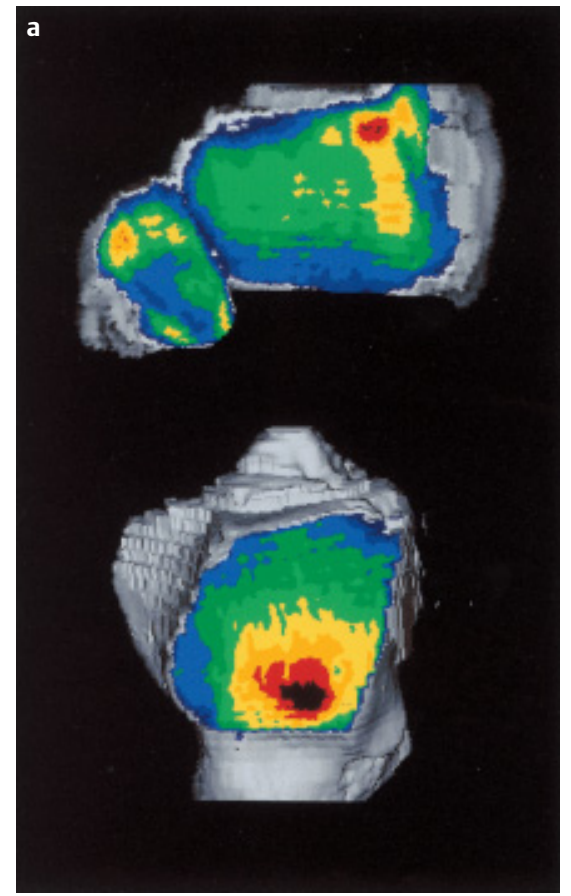

Abb.4au.b Flächenhafte Verteilung der subchondralen Mineralisierung des oberen Sprunggelenks (die Malleolengabel ist jeweils nach oben geklappt). Oben ist jeweils die tibiofibulare Gelenkfläche, unten die talare Gelenkfläche dargestellt. (a) monozentrisches Verteilungsmuster, das üblicherweise bei $\mathrm{Ab}$ flachung der Trochleaführungsrinne zu be-

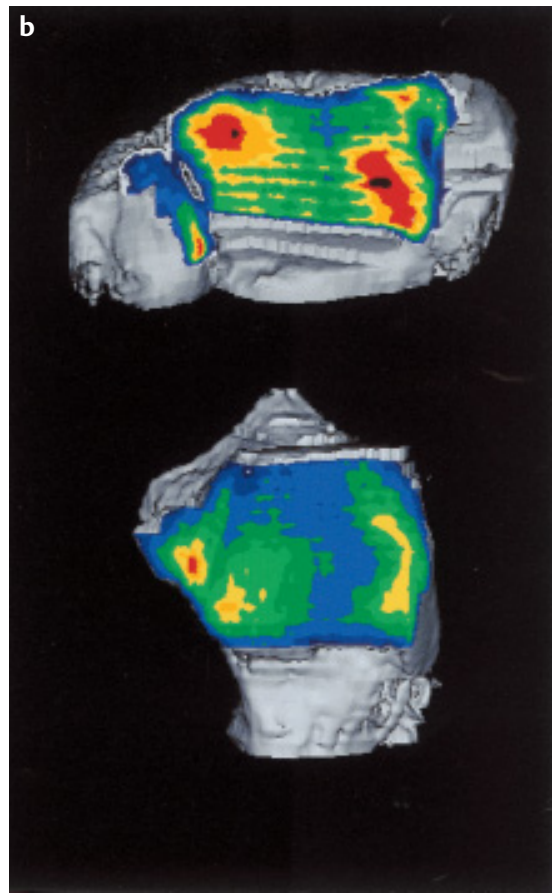

obachten ist, etwa beim älteren Menschen mit degenerativen Verschleißerscheinungen. (b) bizentrisches Verteilungsmuster mit je einem kantennahen Dichtemaximum der medialen und lateralen korrespondierenden Gelenkflächen entsprechend dem häufigsten Verteilungsmuster beim jungen Menschen.

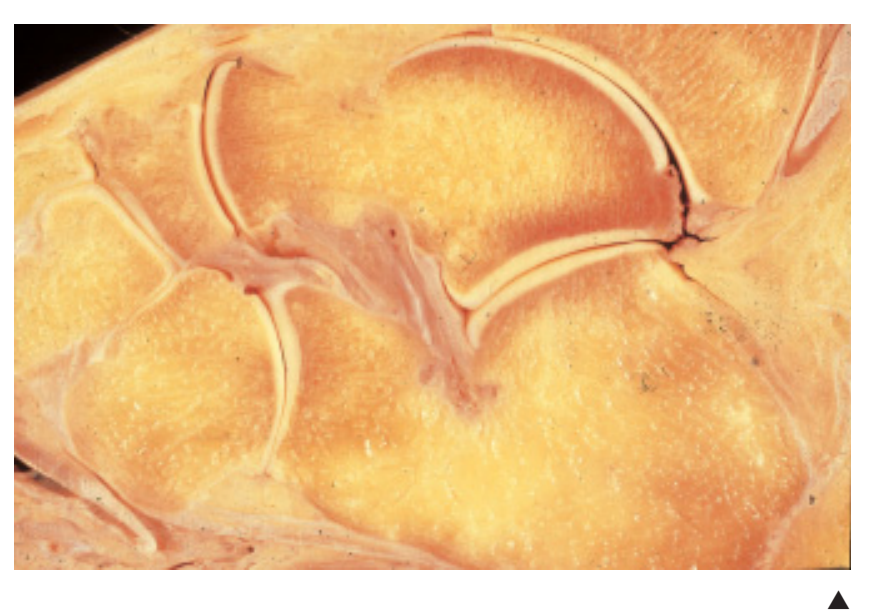

Das Prinzip der physiologischen Inkongruenz sorgt somit für eine bessere Durchwalkung des Gelenkknorpels und für eine günstigere Lastverteilung im Rahmen der Kontaktflächenvergrößerung [16]. Die subchondrale Dichteverteilung kann somit als biologisches Korrelat der individuellen Langzeitbeanspruchung herangezogen werden. Die Darstellung und Quantifizierung der subchondralen Knochendichte ist heute mittels Computertomographie und Osteoabsorptiometrie am Lebenden möglich $[16,17]$.
Abb. 5 Sagittalschnitt durch ein Präparat des ren und des hinteren unteren Sprunggelenks (Subtalargelenk) sowie des vorderen unteren Sprunggelenkes (Chopartgelenklinie = Talonavikular- und Kalkaneokuboidgelenk). menschlichen Fußes mit Darstellung des obe- 


\section{Unteres Sprunggelenk (USG) und Fuß}

Der Talus artikuliert mit dem unter ihm liegenden Kalkaneus und dem vor ihm liegenden Navikulare, der Processus anterior calcanei wiederum mit dem Kuboid, so dass wir ein hinteres unteres Sprunggelenk (Subtalargelenk) und ein vorderes unteres Sprunggelenk (entsprechend dem Chopartgelenk) unterscheiden können (Abb.5). Der Taluskopf liegt in einer fast kugeligen Gelenkhöhle so dass das Talonavikulargelenk aufgrund gewisser morphologisch-funktioneller Analogien als „Acetabulum pedis“ bezeichnet wird [7].

Zum besseren Verständnis der komplexen funktionellen Abläufe bei Bewegungen des Fußes ist es zweckmäßig, den Abschnitt des Fußskeletts distal des Talus als subtalare Platte (Abb. 2) zu charakterisieren.

In dieser subtalaren Platte finden weitere Bewegungen, wie die Pro- und Supination, eine $\mathrm{Ab}$ - und Adduktion des Vorfußes sowie die Plantarflexion/Dorsalextension im Chopartgelenk statt. Zum besseren Verständnis der Mechanik des Fußes kann man eine mediale und laterale Fußsäule bzw. Route des Kraftflusses unterscheiden (Abb. 6). Die schräge Stellung der Achse des USG bewirkt eine Übersetzung der Rotation des Unterschenkels in

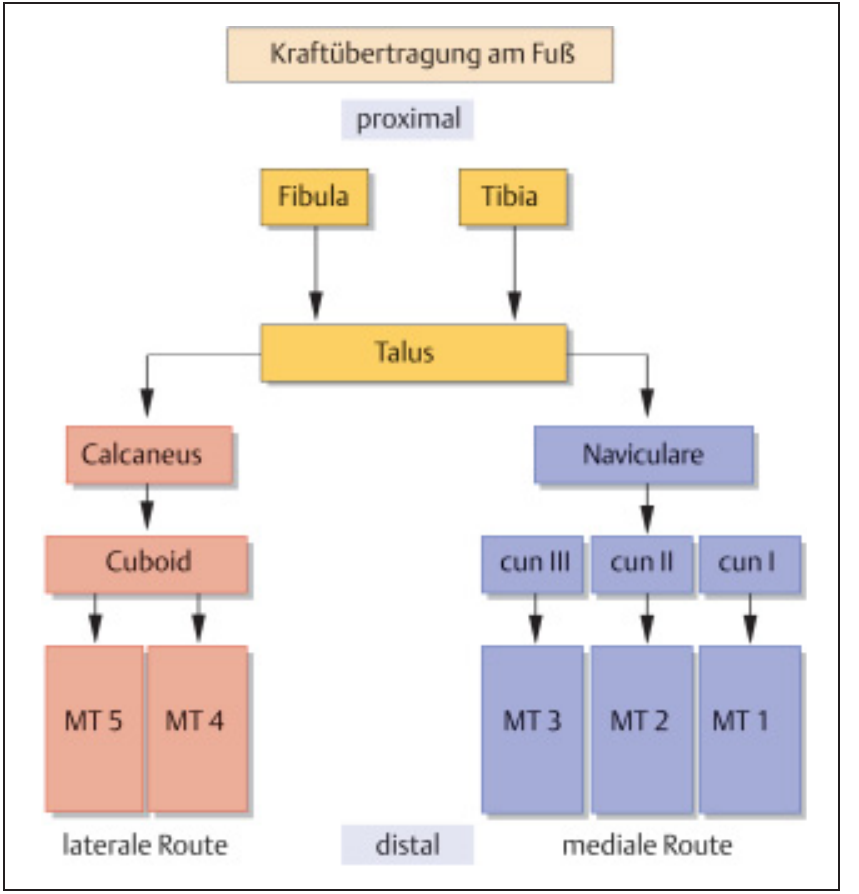

Abb. 6 2-SäulenModell der Kraftüberleitung am Fuß (mediale und laterale Route). Beide Säulen sind eng funktionell aneinander gekoppelt. eine Drehbewegung des Fußes um seine Längsachse. Dies erlaubt es dem Vorfuß auf dem Boden zu bleiben. Bei feststehendem Vorfuß bewirkt die Rotation des Unterschenkels neben der In- und Eversion des Rückfußes eine Verwringung in der subtalaren Fußplatte.

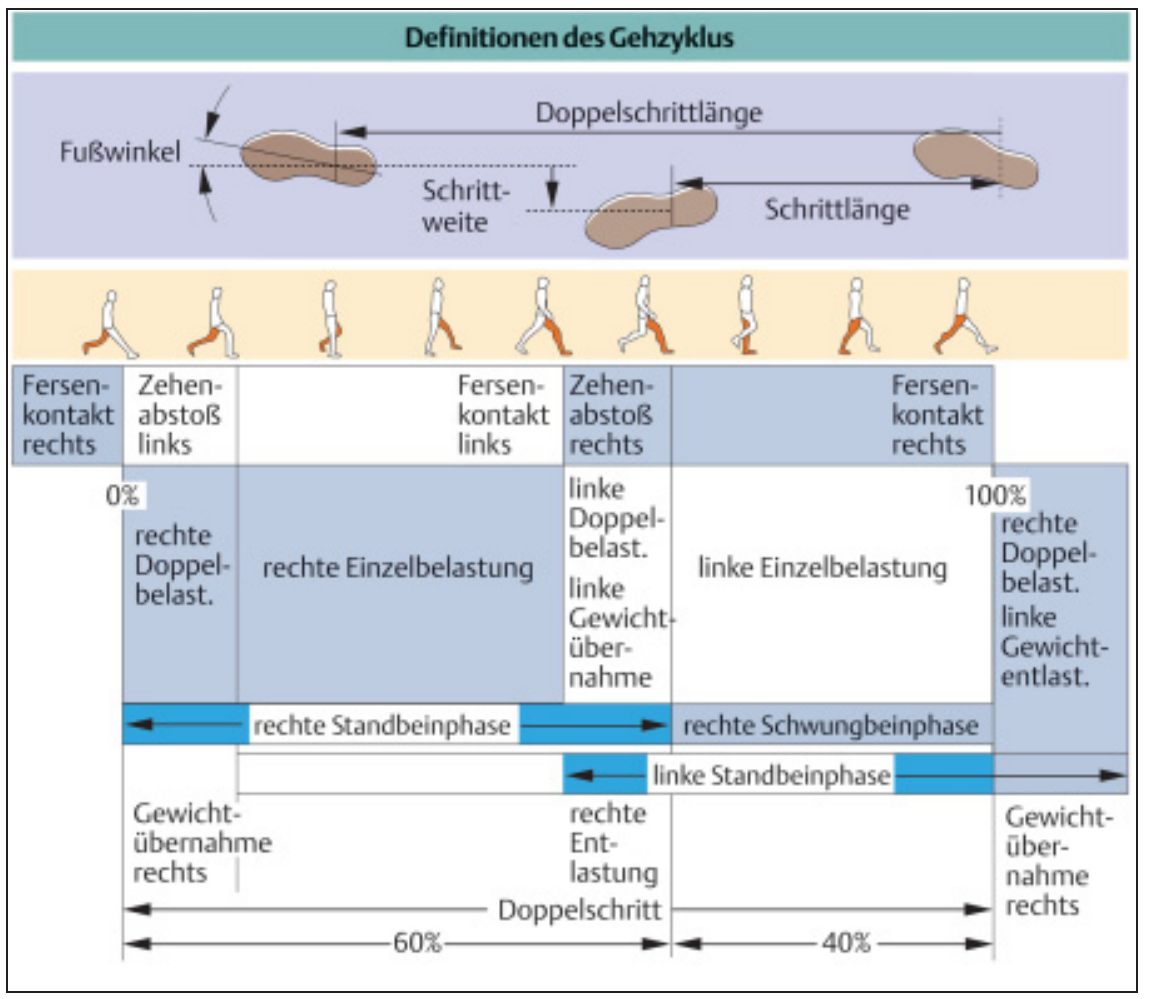

Die Form der subtalaren Fußplatte wird wesentlich durch den Spannungszustand der Plantaraponeurose bestimmt.

Die Plantaraponeurose entspringt am Kalkaneus und strahlt in den plantaren Beugeapparat ein.

Auch im unteren Sprunggelenk lässt sich eine charakteristische Verteilung der subchondralen Knochendichte nachweisen; eine hohe Mineralisierung findet sich entlang der lateralen Kanten der einander zugewandten Flächen des Talokalkanealgelenks. In den beiden Gelenkflächen des Talonavikulargelenks finden sich 2 korrespondierende Dichtemaxima [17], von denen die Dichte zu den Rändern hin konzentrisch abfällt. Auch an diesen Gelenken ist das Prinzip der physiologischen Inkongruenz demonstrierbar [17].

\section{Ganganalyse und Funktion des Fußes}

Die vollständige Beschreibung des menschlichen bipedalen Ganges beinhaltet eine Fülle von Informationen, die neben kinematischen Parametern (Ortskoordinaten, Winkelverläufe und deren Veränderungen) und kinetischen Parametern (z.B. Bodenreaktionskraft, dynamische Druckverteilungsmessung unter dem Fuß) auch dynamische neuromuskuläre Parameter umfasst $[4,6,7,11$, 21,22] (z.B. dynamisches EMG).

4 Abb. 7 Definitionen des Gangzyklus. 

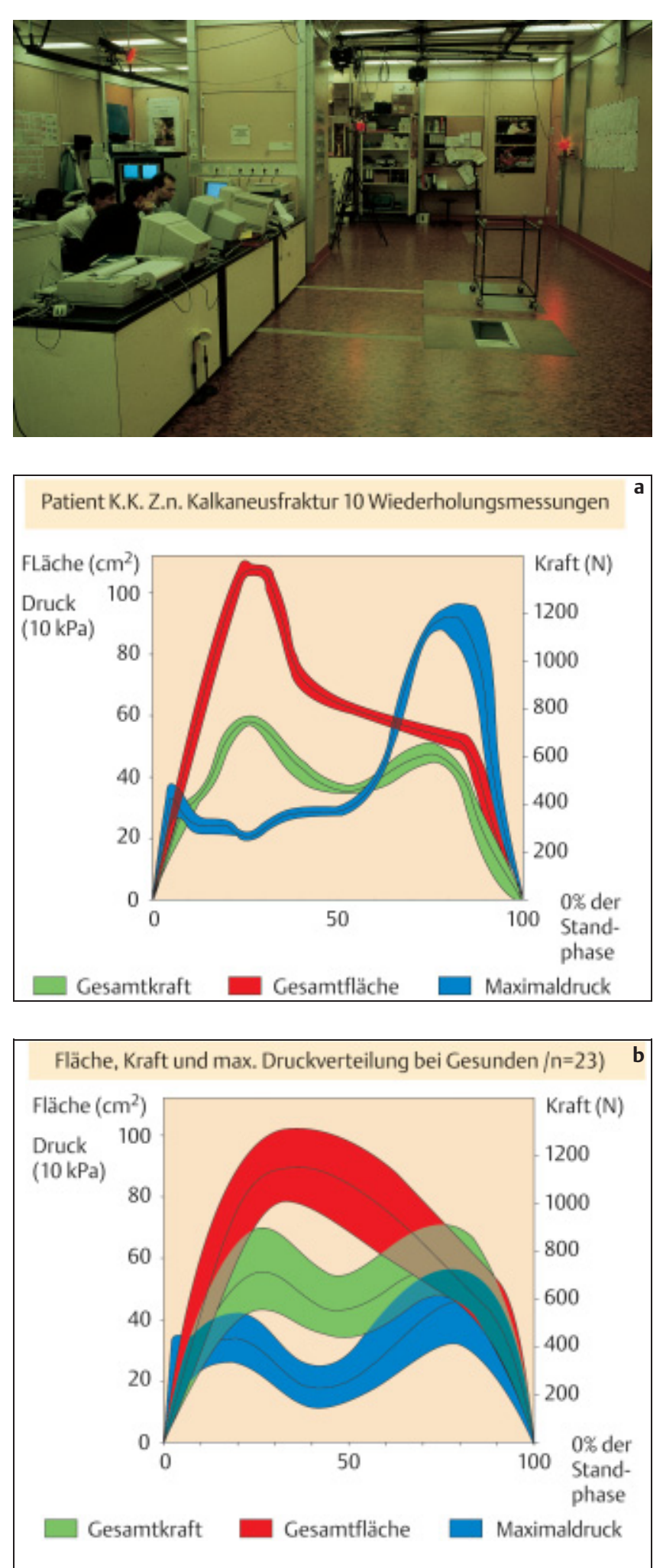

Abb.9 Verlauf der 3 Basisparameter der dynamischen Druckverteilungsmessung (Kraft, Fläche und Maximaldruck) während der Standphase des Gangzyklus. (a) in einem Kollektiv gesunder Probanden. (b) bei einem Patienten nach operativ rekonstruierter Kalkaneusfraktur (10 Wiederholungsmessungen).
Abb. 8 Ganglabor mit optoelektronischem Video-Prozessorsystem, 2 Kraftmessplatten und einer Plattform zur Druckverteilungsmessung (der Laufsteg mit den integrierten Plattformen ist üblicherweise verdeckt).

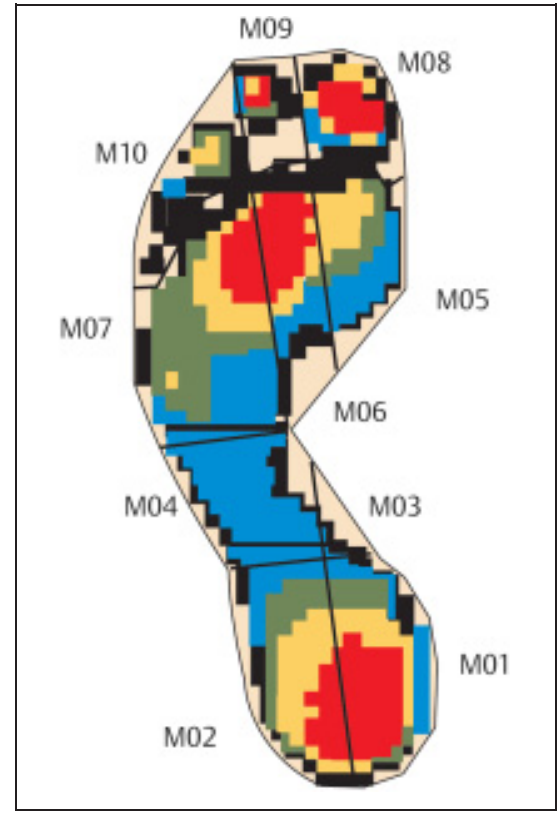

Abb.10 Dynamische Druckverteilungsmessung (dynamische Pedographie): Einteilung des summativen Maximaldruckbildes eines Abrollzyklus in 10 „regions of interest“ (Masken 01-10 (EMED SF-4, Novel GmbH, München).

Mehr denn je bieten die heutigen messtechnischen Verfahren und Methoden der Informationsverarbeitung Möglichkeiten, Daten anzuhäufen ohne hinsichtlich des eigentlichen Untersuchungszwecks verbindliche Aussagen treffen zu können.

Im klinischen Gebrauch ist daher die zugrunde liegende Fragestellung entscheidend für die Wahl eines spezifischen ganganalytischen Untersuchungsverfahrens, dessen Einsatz Vorteile gegenüber der rein klinischen Beurteilung bringen soll. Eine zeitgemäße klinische Ganganalyse soll somit Erkenntnisse liefern, die der erfahrene Kliniker nicht direkt beobachten kann. Die Resultate sollen so formuliert sein, dass sie in Einklang mit einem klinischen Konzept zu bringen sind [4]. Die klinische Fragestellung wird auch darüber entscheiden, auf welche relevanten Messparameter wir die Menge der Basisdaten einer funktionellen Untersuchung reduzieren können [21] (Parameterselektion). 

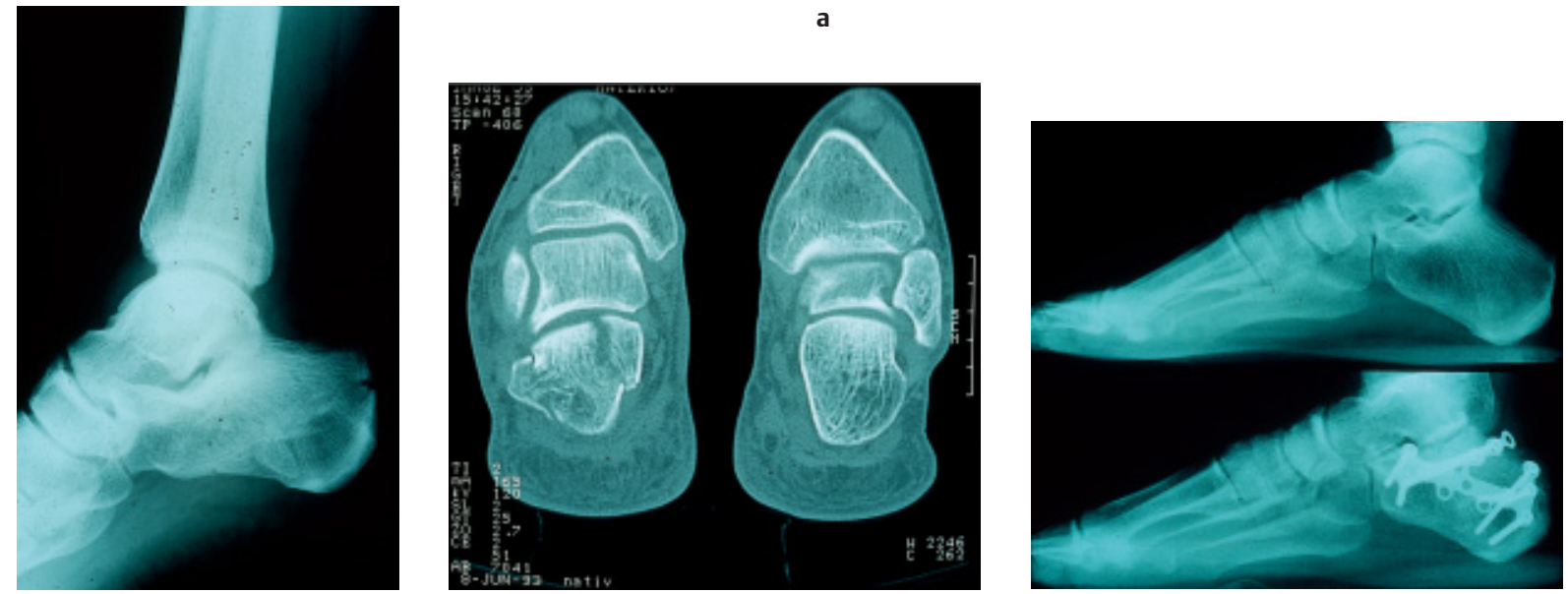

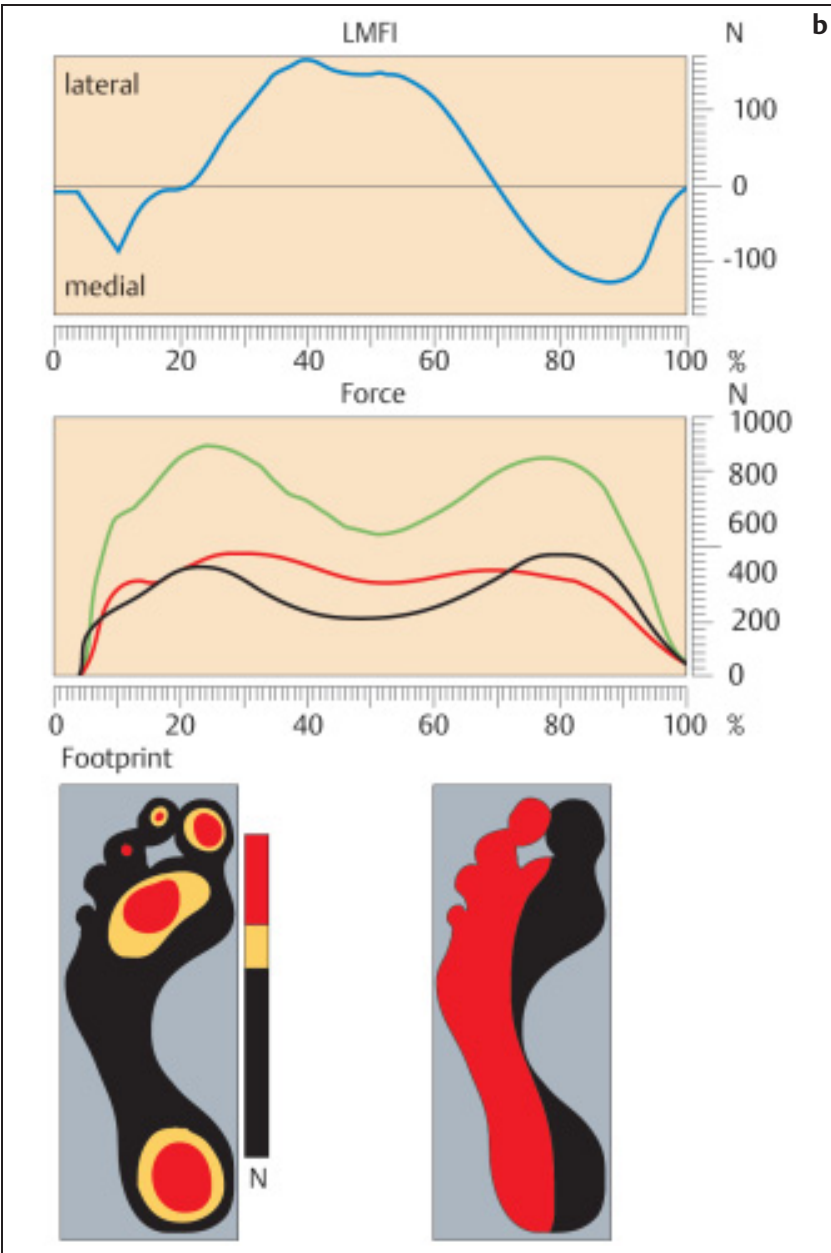

Abb.11 Patient mit intraartikulärer Kalkaneusfraktur Typ Sanders II B rechts mit ausgezeichnetem klinischen Resultat (Beschwerdefreiheit). (a) Unfallröntgenbild Fersenbein seitlich, eine CT-Sequenz beider Füße und seitliche Belastungsaufnahmen beider Füße im Stehen 6 Monate nach OP. (b) medio-lateraler Kraftindex, Fläche, Kraft und Maximal-

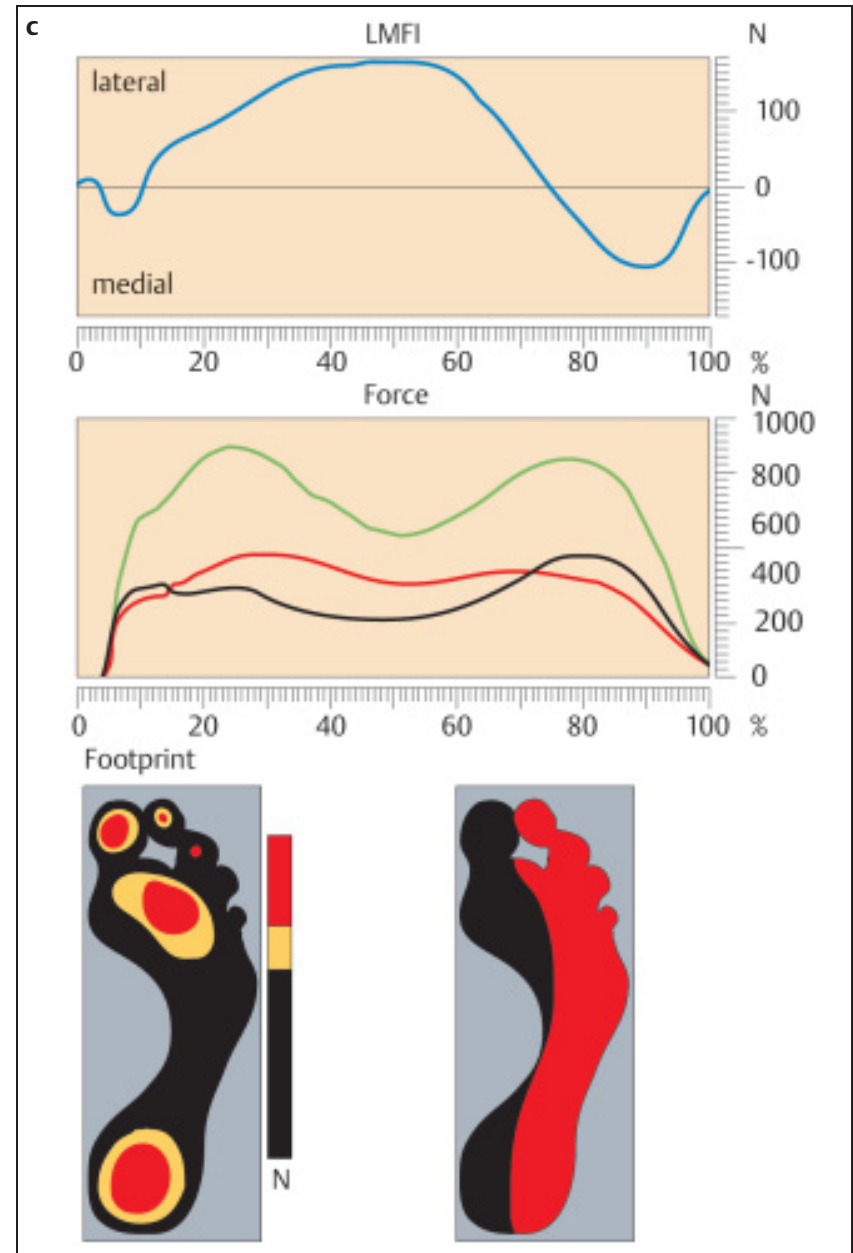

druckbild eines Abrollzyklus der unverletzten Extremität. (c) medio-lateraler Kraftindex, Fläche, Kraft und Maximaldruckbild eines Abrollzyklus der verletzten Extremität. (grün $=$ Gesamtkraft, schwarz $=$ mediale Teilkraft, rot = laterale Teilkraft) 


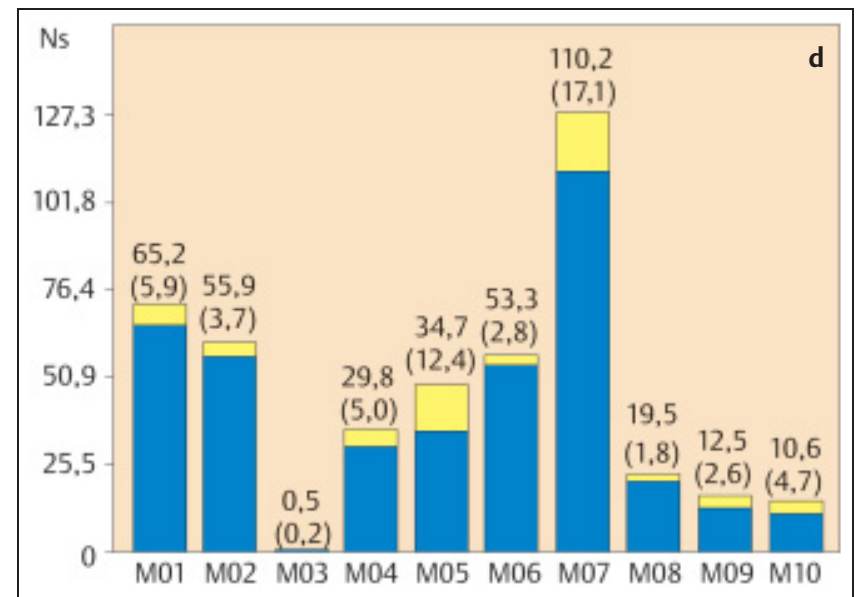

Abb.11 (d) Kraft-Zeit-Integral (Impuls) in den 10 regions of interest der unverletzten Extremität. (e) Kraft-Zeit-Integral (Impuls) in den 10 regions of interest der verletzten Extremität (Standardabweichung in

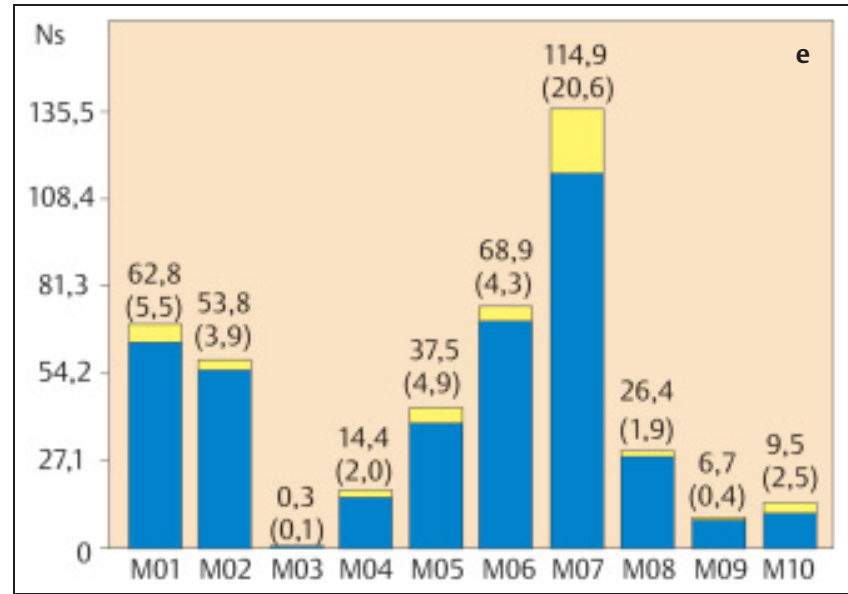

Klammern). $\rightarrow$ sämtliche Parameter zeigen eine fast vollständige Entsprechung (Symmetrie) an beiden Extremitäten (unverletzt versus verletzt).
Grundsätzlich sind vier Anwendungsgebiete definiert, in denen ein technisches Verfahren zur Gangbeurteilung zu einer Problemlösung beitragen kann [4]:

zur Diagnosesicherung

- zur Bewertung eines Schweregrades einer Erkankung/Verletzung (hierunter fallen auch sämtliche Fragestellungen der Qualitätskontrolle bzw. des objektiven funktionellen Vergleichs verschiedener konkurrierender Behandlungsverfahren)

- zur Selektion einer therapeutischen Option und

zur Prognosebewertung.

\section{Ganganalyseverfahren}

\section{Definitionen}

Bei der Ganganalyse wird der Gangzyklus in verschiedene Phasen unterteilt $[6,20\}$ (Abb.7). Grundeinheit ist der Doppelschritt (=100\%), gerechnet vom Fersenkontakt einer Extremität bis zum erneuten gleichseitigen Fersenkontakt. Zeitliche Asymmetrien (z.B. Standphasendauer einer gesunden und einer verletzten Extremität) können an ein und demselben Patienten bereits durch einfache Messungen der Bodenkontaktzeiten über entsprechende Kontaktschalter am Schuh bzw. der Fußsohle ermittelt werden und orientierende Hinweise auf Störungen des Abrollmusters geben [6].

\section{Kinetisch-kinematische Untersuchungen}

Kraftmessplattformen wurden während der letzten beiden Dekaden häufig eingesetzt, um die Bodenreaktionskräfte als Summe aller Druckkräfte zwischen Fußsohle und Boden zu analysieren. Hierbei werden die 3 Raumkomponenten der Bodenreaktionskraft gemessen, der Kraftvektor rekonstruiert und sein Angriffspunkt an der Auftrittsfläche bestimmt $[6,7,21]$ („Ganglinie“).

Neben zahlreichen Parametern der Kontaktphase (z.B. Brems- oder Beschleunigungsimpuls) wurden in der Vergangenheit weitere oft relativ abstrakte Parameter abgeleitet, in der Vorstellung, hiermit Trennfunktionen angeben zu können, die für die Abrollstörung nach definierter Verletzung oder bei bestimmter Grunderkrankung typisch sind $[6,7,11]$. Dies scheiterte überwiegend zum einen wegen der Fehleinschätzung der Bedeutung der Bodenreaktionskraft, zum anderen wegen der fehlenden Ortsauflösung.

Mit Hilfe der Messgrößen der Kraftmessplatten in Kombination mit optoelektronischen Vorrichtungen zur Bestimmung der Ortskoordinaten definierter Körpersegmente lassen sich über entsprechende mechanische Modelle (z.B. der unteren Extremität oder des Fußes) innere Gelenkkräfte- und- momente errechnen $[6,11,12,13,14,22]$. Diese relativ aufwendige Technik ist stets dann erforderlich, wenn auf unterschiedlichen Gelenkniveaus mit komplexen Funktionsstörungen und entsprechenden Kompensations- und/oder Adaptationsmechanismen zu rechnen ist bzw. deren vollstän- dige Erfassung für die Therapieplanung bedeutsam ist (Abb.8). In Verbindung mit dynamischen EMG-Untersuchungen definierter Kennmuskeln wird diese vollständige Form der Ganganalyse üblicherweise bei der Therapieplanung neuroorthopädischer Erkrankungen (z.B. infantile Zerebralparese) angewandt [22]. An den Gelenken des Fußes hat dieses aufwendige Verfahren, u. a. wegen der relativ geringen Bewegungsausschläge im USG und den Gelenken der Fußwurzel und des Vorfußes sowie der limitierten räumlichen Auflösung bei der Verwendung von Oberflächenmarkern, nur bei der Funktionsanalyse des OSG und des Großzehengrundgelenkes erlangt $[1,14]$. In eigenen Untersuchungen konnte mit Hilfe der "full-gait-analysis“ nach komplexen Verletzungen des Fußes nachgewiesen werden, dass auf allen untersuchten Gelenkebenen - USG, OSG Knie, Hüfte und Becken - Ausgleichsbewegungen stattfinden, die jedoch erhebliche interindividuelle Varianz aufweisen [12]. Eine korrekte Aussage über die Pathobiomechanik ist somit nur möglich, wenn alle Gelenkebenen der unteren Extremität untersucht werden [13].

\section{Dynamische Druckverteilungsmessung}

Bei Erkrankungen oder nach Verletzungen des Fußes wurden während der letzten 15 Jahre dynamische Druckverteilungsmessungen (dynamische Pedographie) zunehmend häufig eingesetzt, die eine wenig aufwendige Erfassung der Vertikalkomponente der Bodenreaktionskraft mit hoher örtlicher (2-8 Sensoren $\left./ \mathrm{cm}^{2}\right)$ und zeitlicher $(50-100 \mathrm{~Hz})$ Auflösung erlauben $[2,3,7,11]$. Neben tra- 

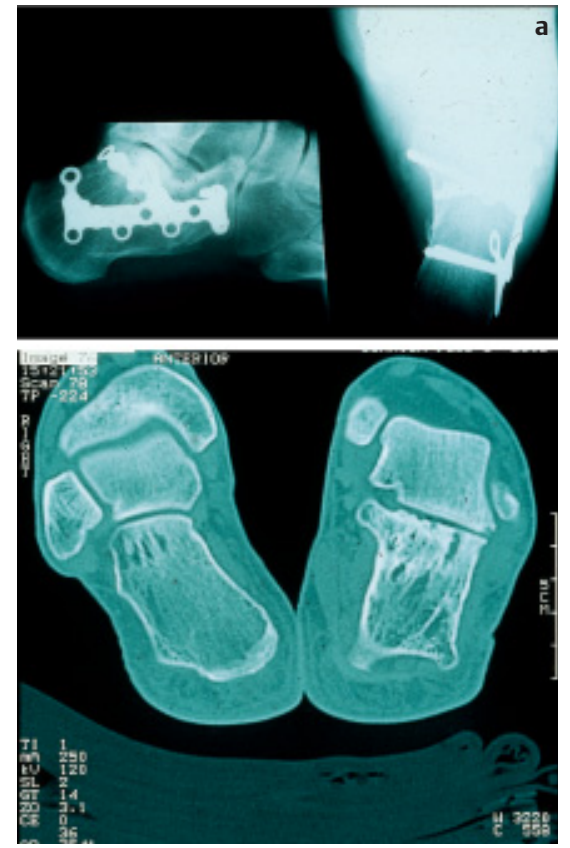

Abb.12 12 Patienten nach intraartikulärer Kalkaneusfraktur Typ Sanders III AC links mit belastungsabhängigen Beschwerden beim Gehen. (a) Röntgenbild Fersenbein seitlich nach operativer Rekonstruktion, Manifestation posttraumatischer Arthrosezeichen im hinteren Subtalargelenk im CT nach Implantatentfernung 8 Monate postoperativ. (b) medio-lateraler Kraftindex, Fläche, Kraft und Maximaldruckbild eines Abrollzyklus der unverletzten Extremität. (c) medio-lateraler Kraftindex, Fläche, Kraft und Maximaldruckbild eines Abrollzyklus der verletzten Extremität. (grün = Gesamtkraft, schwarz = mediale Teilkraft, rot $=$ laterale Teilkraft)

ditionellen Plattformmessungen besteht auch die Möglichkeit der Im-Schuh-Messung mittels instrumentierter Einlegesohlen (z.B. standardisierte Testung auf dem Laufband). Dies erlaubt es auch, Sportschuhkonstruktionen, Einlagen, Orthesen oder spezielle therapeutische
Schuhzurichtungen hinsichtlich ihrer Auswirkungen unter Feldbedingungen dynamisch zu untersuchen. Der Messvorgang ist einfach und rasch durchführbar, also auch bei klinischen Verlaufskontrollen ohne größeren Aufwand praktikabel. Die Messresultate werden jedoch ganz entscheidend von der Aufnehmercharakteristik bestimmt; eine akzeptable $\mathrm{Ge}$ nauigkeit (Messfehler $>5 \%$ ) und eine Langzeitstabilität der Kalibrierung weisen nur kapazitive Messsysteme auf $[1,7,11]$. Bestimmt werden die Basisparameter Druck, Zeit, belastete Fläche unter dem Fuß beim Gehen (Abb.9). Hieraus lassen sich die Gesamtkraft und weitere abgeleitete Parameter (z.B. der Impuls unter dem Fuß beim Gehen) errechnen [11]. Mit Hilfe statistischer Verfahren bieten funktionelle Scores, die oftmals mehrere Parameter der dynamischen Pedographie umfassen, die Option eine Gangstörung quantitativ zu objektivieren [11 -
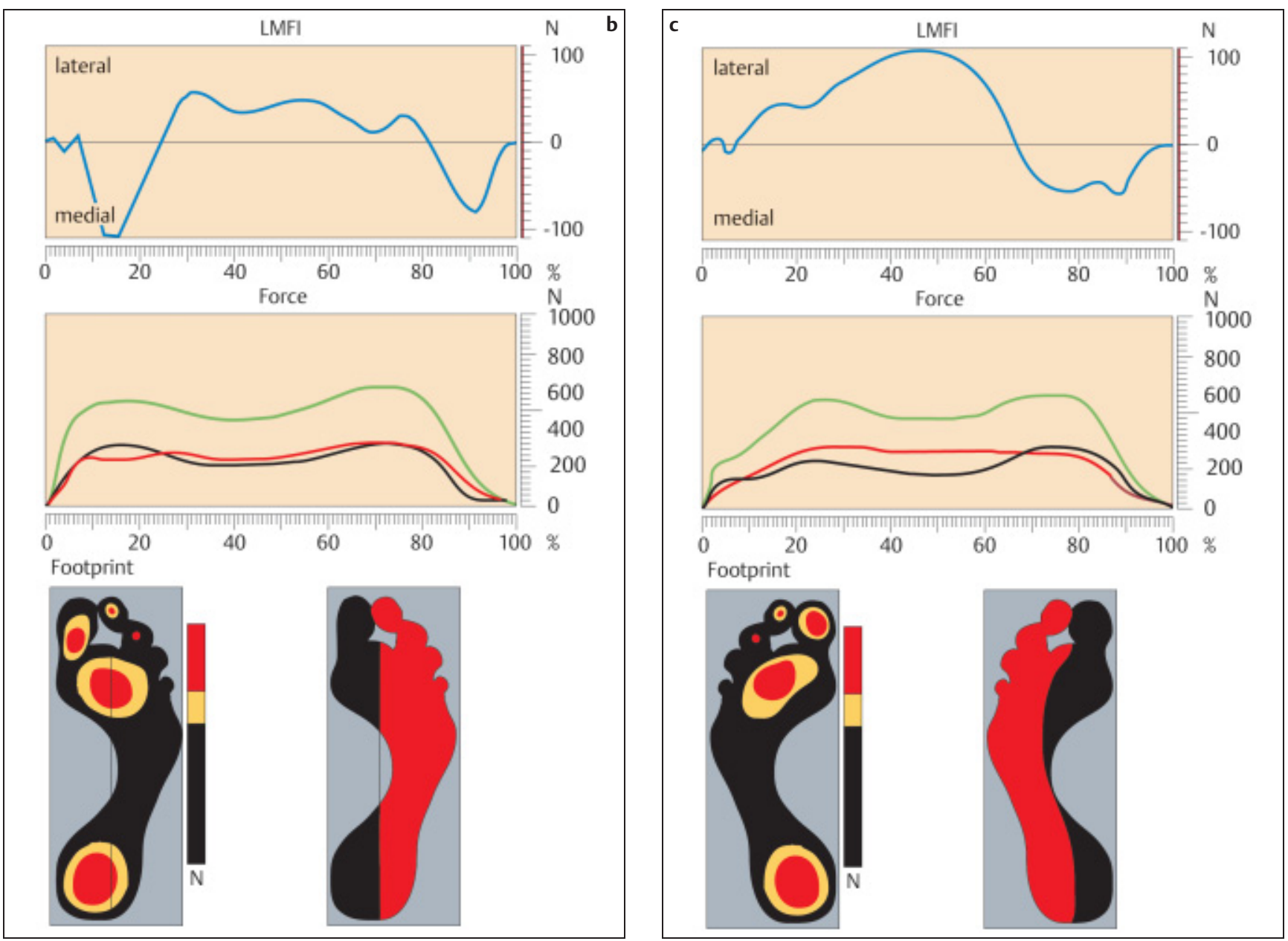


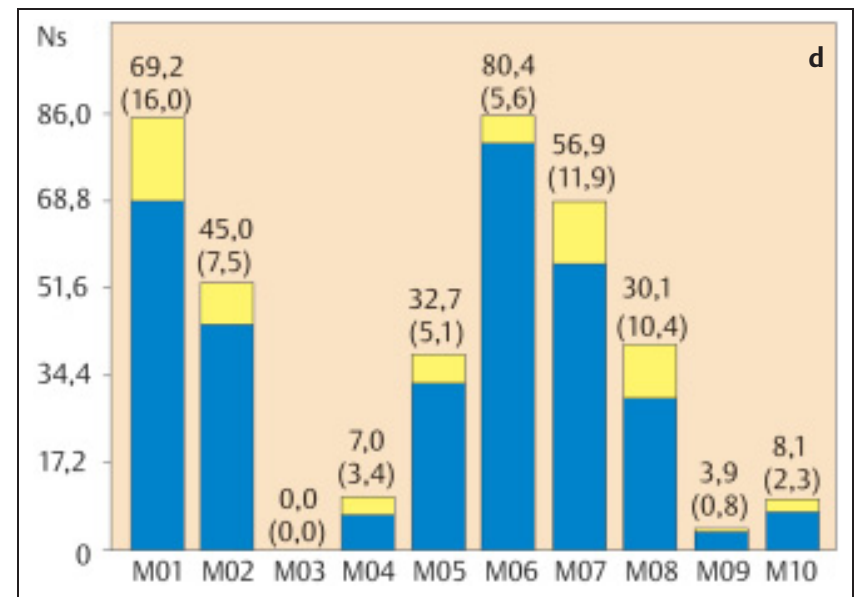

Abb.12 (d) Kraft-Zeit-Integral (Impuls) in den 10 regions of interest der unverletzten Extremität. (e) Kraft-Zeit-Integral (Impuls) in den 10 regions of interest der verletzten Extremität (Standardabreichung in

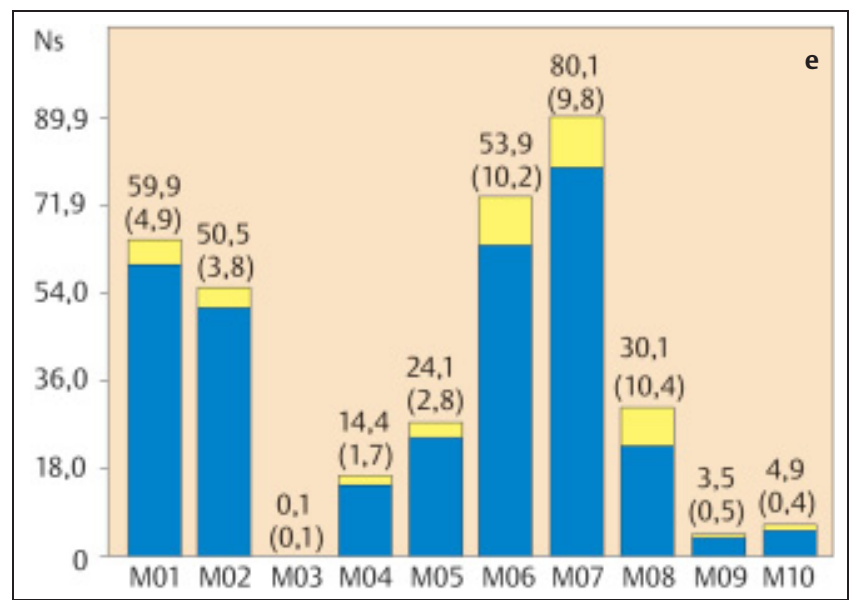

Klammern). $\rightarrow$ deutliche Asymmetrie der Parameter der Druckverteilungsmessung (unverletzt versus verletzt), Shift der Belastung nach lateral am vormals verletzten Fuß.
13]. Es ist jedoch nicht zulässig, anzunehmen, dass für unterschiedliche klinische Fragestellungen stets die gleiche Zahl und Kombination von Parametern die Voraussetzung zur optimalen Diskrimination funktioneller Störungen bietet. Ist eine optimale Parameterauswahl für eine spezifische klinische Applikation getroffen worden, bieten Softwarelösungen die Möglichkeit zur zeitsparenden Auswertung der Funktionsprüfung.

Die Parameter können nicht nur unter der gesamten Kontaktfläche des Fußes beim Abrollvorgang, sondern auch in (untersucher-) definierten Teilregionen („regions of interest“) ermittelt werden (Abb.10). Trotz gewisser Weichteilverschiebungen beim Abrollvorgang wird somit eine Zuordnung zu anatomischen Regionen des Fußes möglich.

Rückschlüsse von kinetischen Messgrößen der dynamischen Pedographie auf kinematische Prozesse oberhalb der USGEbene sind jedoch nicht zu ziehen $[12,13]$.

Probleme bereiten nach wie vor die Referenzgrößen der jeweiligen Parameter. Der „normale“ Gang ansonsten beschwerdefreier „fußgesunder“ Probanden weist erhebliche interindividuelle Varianz hinsichtlich der jeweiligen Messgrößen auf (vgl. Abb.9a). Die Reproduzierbarkeit intraindividueller Wiederholungsmessungen ist erstaunlich hoch (Abb.9b), so dass in Situationen, wo ein klassischer Vorher-Nachher-Vergleich z.B. vor und nach Korrekturosteotomie am I. Metatarsalstrahl) nicht möglich ist, intraindividuelle Vergleichsmessungen,
z.B. zwischen verletzter und unverletzter Extremität, sich als vorteilhaft und relativ robust gegen Störeinflüsse erwiesen haben [11-13].

Eines des frühesten klinischen Applikationsgebiete der dynamischen Druckverteilungsmessung lag bei der Erfassung des Risikoprofils von Patienten mit diabetischer Neuropathie und somit bei der Früherkennung und Prophylaxe plantarer diabetischer Ulzera [5]. Mit Hilfe der dynamischen Pedographie lassen sich die Schweregrade von Abrollstörungen bei definierten orthopädischen Erkrankungen (z.B. Hallux valgus, Hallux rigidus, Dysfunktion der Tibialis posterior-Sehne, Rheumatoide Arthritis) quantitativ erfassen und die Effektivität therapeutischer Maßnahmen objektivieren [7,11].

Mit Hilfe der dynamischen Druckverteilungsmessung lässt sich das Ausmaß einer Gangstörung nach definierter Verletzung oder spezifischer Behandlung mit Hilfe des intraindividuellen Vergleichs (Beurteilung der Symmetrie) mit hoher Auflösung erfassen [11-13] (vgl. Abb.11 u.12).

Während Patienten mit funktionell vollständig ausgeheilter Verletzung idealerweise eine intraindividuelle Symmetrie von Kennparametern aufweisen, die nicht den Kontrollbereich der Parameter bei gesunden Probanden überschreitet (Abb.11), zeigen Patienten mit klinischer Beschwerdesymptomatik (z.B. nach OSGFraktur, Kalkaneusfraktur, Verletzungen der Chopart- und/oder Lisfrancgelenklinie) typische Störungen, wie den nach lateral gerichteten Lasttransfer distal des
Subtalargelenks $\quad[2,3,12,15] \quad$ (Abb.12), der eine erhebliche Störung des Abrollverhaltens bedeutet und sich mit progredienter funktioneller Dekompensation in einem hinkenden Gangbild äußert. Diese Erkenntnisse aus ganganalytischen Untersuchungen lassen sich mit Hilfe von In vitro-Modellen nachvollziehen bzw. anhand der typischen Pathobiomechanik erklären [19].

Somit lässt sich mit Hilfe der dynamischen Pedographie ein Zusammenhang zwischen Morphologie und Funktion des Fußes herstellen: Manifeste Fehlstellungen des Fußes (z.B. Varus- oder Valgusabweichungen, Höhen-, Längenverlust oder die Verbreiterung des Rückfußes nach Kalkaneusfraktur) lassen sich in ihrer Auswirkung auf die Gehfunktion objektiv bewerten [15].

Die mit Hilfe der dynamischen Pedographie gewonnenen Erkenntnisse bieten sich als Instrument der Qualitätskontrolle an, lassen sich in die OP-Planung und -taktik einbeziehen und können auch einen Baustein für die Indikationsstellung zu korrektiven Maßnahmen darstellen.

\section{Ausblick}

Unter den genannten Aspekten dürfte die Zukunft der klinisch anwendbaren Ganganalysen des Fußes weniger auf dem Sektor der technischen Innovation oder Verbesserung der Messprinzipien bzw. der steigenden Komplexität der Auswertesoftware liegen. Priorität hat die Parametrisierung, also die Reduktion der Information auf die relevanten Daten, und 
die Validierung der ganganalytischen Kenngrößen an geeigneten klinischen Kollektiven. Eine allgemeine Akzeptanz ganganalytischer Techniken im klinischen Alltag ist wesentlich davon abhängig, inwieweit eine Allgemeingültigkeit jener Erkenntnisse nachgewiesen werden kann, die bislang an speziellen Untersuchungsmodellen nachgewiesen wurden

\section{Literatur}

${ }^{1}$ Alexander IJ, Chao EYS, Johnson KA. The assessment of dynamic foot-to-ground contact forces and plantar pressure distribution: a review of the evolution of current techniques and clinical applications. Foot Ankle Int 1990; 11: $152-167$

${ }^{2}$ Becker HP, Rosenbaum D, Zeithammel G Gerngroß H, Claes L. Gait pattern analysis after ankle ligament reconstruction (modified Evans procedure). Foot Ankle Int 1994; 15 477-482

${ }^{3}$ Becker HP, Rosenbaum D, Kriese T, Gerngroß $\mathrm{H}$, Claes L. Gait asymmetry following successful surgical treatment of ankle fractures in young adults. Clin Orthop 1995; 311: $262-269$

${ }^{4}$ Brand RA. Can biomechanics contribute to clinical orthopaedic assessments? J Biomech 1987; 19: 453-457

${ }^{5}$ Cavanagh PR, Simoneau GG, Ulbrecht JS. Ulceration, unsteadiness, and uncertainty: the biomechanical consequences of diabetes mellitus. J Biomech 1993; 26 Suppl I: $23-40$

${ }^{6}$ Chao EYS. Gait analysis: a survey. In: Bergmann G, Kölbel R, Rohlmann A (eds.): Biomechanics: basic and applied research. $M$. Nijhoff, Dordrecht, 1987; 34-49
7 Debrunner HU, Jacob HAC. Biomechanik des Fußes. 2.Aufl., Ferdinand Enke, Stuttgart 1998

${ }^{8}$ Hennig EM, Milani TL. Die Dreipunktunterstützung des Fußes - eine Druckverteilungsanalyse bei statischer und dynamischer Belastung. Z Orthop 1993; 131: 279-284

${ }^{9}$ Kapandji IA. Funktionelle Anatomie der Gelenke. Band 2: Untere Extremität. Ferdinand Enke, Stuttgart, 1985

${ }^{10}$ Ledermann M, Cordey J. Messmethodik und Standardwerte zur Untersuchung der Dynamik der Syndesmose beim Menschen. In: Hackenbroch MH, Refior HJ, Jäger M, Plitz W (Hrsg): Funktionelle Anatomie und Pathologie des Sprunggelenks. G. Thieme, Stuttgart, 1984

${ }^{11}$ Mittlmeier Th, Morlock MM. Statische und dynamische Belastungsmessungen am posttraumatischen Fuß. Orthopäde 1991; 20: $22-32$

${ }^{12}$ Mittlmeier Th, Morlock MM, Kollmitzer J Zwick E-B, Lob GC. Efficiency of gait measurement after complex foot trauma. Foot Ankle Surg 1996; 2: 197-208

${ }^{13}$ Mittlmeier Th. Aussagekraft der Ganganalyse bei bestehenden posttraumatischen Fehlstellungen. In: Strecker W, Keppler P, Kinzl L (Hrsg.): Posttraumatische Beindeformitäten. Analyse und Korrektur. Springer, Berlin, 1997; 111 - 119

${ }^{14}$ Morlock MM, Nigg BM. Theoretical considerations and practical results on the influence of the representation of the foot for the estimation of internal forces with models. Clin Biomech 1991; 6: 3-13

${ }^{15}$ Mittlmeier Th, Morlock MM, Hertlein $\mathrm{H}$ Fäßler M, Mutschler W, Bauer G, Lob G. Analysis of morphology and gait function following intraarticular calcaneal fracture. J Orthop Trauma 1993; 7: $303-310$

${ }^{6}$ Müller-Gerbl M. Anatomie und Biomechanik des oberen Sprunggelenks. Orthopäde 2001; 30: 3-11
17 Putz R, Müller-Gerbl M. Funktionelle Anatomie des Fußes. Orthopäde 1991; 20: 2-10

${ }^{18}$ Procter P, Paul JP. Ankle joint biomechanics. J Biomech 1982; 15: 627-634

${ }^{19}$ Rosenbaum D, Bauer G, Augat P, Claes L. Calcaneal fractures cause a lateral load shift in Chopart joint contact stresses and plantar pressure pattern in vitro. J Biomech 1996; 29: $1435-1443$

${ }^{20}$ Sarrafian JB. Anatomy of the foot and ankle. JB Lippincott, Philadelphia, 1983

${ }^{21}$ Stüssi E. Was heißt Ganganalyse? Swiss Med 1987; 9: 8-13

${ }^{22}$ Winter DA. Biomechanics and motor control of human movement. 2nd ed. J. Wiley, Toronto, 1990

Prof. Dr. med. Thomas Mittlmeier kommissarischer Leiter der Abteilung für Unfallchirurgie

Chirurgische Klinik

der Universität Rostock

Schillingallee 35

18055 Rostock

Prof. Dr. med. Magdalena Müller-Gerbl

Anatomische Anstalt

Ludwig-Maximilians-Universität

München

Pettenkofer Straße 11

86336 München 